\title{
On Delta-suitable elements in algebraic lattices
}

\author{
Vanja Stepanovića ${ }^{\text {, Andreja Tepavčević }}{ }^{b}$ \\ ${ }^{a}$ University of Belgrade, Faculty of Agriculture, Nemanjina 6, 11080, Beograd - Zemun \\ ${ }^{b}$ University of Novi Sad, Faculty of Science, Department of Mathematics and Informatics, Trg Dositeja Obradovica 4, 21000 Novi Sad
}

\begin{abstract}
In this paper we introduce new methods and results that can lead to the solution of a longstanding open problem of the representation of algebraic lattices by weak congruences. Here known criteria for $\Delta$-suitable elements in algebraic lattices are generalized.
\end{abstract}

\section{Introduction}

An important lattice determining the structure of an algebra is the weak congruence lattice, i.e., the lattice consisting of all the weak congruences of that algebra, ordered by inclusion. That lattice contains the interval sublattice isomorphic to the subuniverse lattice of the algebra, as well as the interval lattice equal to the congruence lattice of the algebra. Even more, it contains the congruence lattices of all the subalgebras of the algebra. The weak congruence lattice of an algebra is an algebraic lattice, in which the diagonal element $\Delta$ plays a special role. It is the smallest congruence of the algebra and the filter $\uparrow \Delta$ in the weak congruence lattice is equal to the congruence lattice of the algebra. The ideal $\downarrow \Delta$ is isomorphic to the subalgebra lattice of the algebra.

Some of the best known representations of algebraic lattices by different algebraic objects are representations by subuniverses and by congruences of an algebra (solved within Birkhoff-Frink and Grätzer-Schmidt theorems). In paper [6], W. Lampe gives a survey of some recent results and problems on congruence lattice representations.

The problem of representation of an algebraic lattice by the weak congruence lattice of an algebra is still open. Given an algebraic lattice $\mathcal{L}=(L, \vee, \wedge)$ and $a \in L$, the problem is whether there is an algebra $\mathcal{A}$, such that the weak congruence lattice of $\mathcal{A}$, denoted by $C w \mathcal{A}$, is isomorphic to $\mathcal{L}$ under an isomorphism mapping $\Delta$ to $a$.

If that is the case, we say that $a$ is a $\Delta$-suitable element of $\mathcal{L}$. Since $\Delta$ is always a codistributive element in $C w \mathcal{A}$, we conclude that the codistributivity is a necessary condition for an element $a$ of an algebraic lattice to be $\Delta$-suitable. There are also other conditions that a $\Delta$-suitable element should fulfill. Some known necessary conditions for a codistributive element of an algebraic lattice to be $\Delta$-suitable are given in [13] and in [10] and a detailed survey of known results is given in [8].

This paper aims to generalize some of these criteria.

2010 Mathematics Subject Classification. Primary 08A30; Secondary 08A05

Keywords. Weak congruences, $\Delta$-suitable elements, representation of lattices

Received: 09 September 2011; Accepted: 15 December 2011

Communicated by Miroslav Ćirić

Research supported by the Ministry of Education and Science of the Republic of Serbia, Grant No. 174013

Email addresses: dunjic_v@yahoo.com (Vanja Stepanović), andreja@dmi .uns.ac.rs (Andreja Tepavčević) 


\section{Preliminaries}

Let $\mathcal{L}=(L, \vee, \wedge)$ be a complete lattice and $a, b \in L$. $a$ is covered by $b$ ( $b$ covers $a)$, denoted by $a<b$ if $a<b$ and $(a, b)=\emptyset$. As usual, the supremum of $X \subseteq L$ in $\mathcal{L}$ is denoted by $\bigvee X$, and the infimum by $\wedge X$.

A filter generated by $a$, is denoted by $\uparrow a$ and the ideal generated by $a$, by $\downarrow a$ :

$$
\uparrow a=\{x \in L \mid x \geqslant a\}, \quad \downarrow a=\{x \in L \mid x \leqslant a\} .
$$

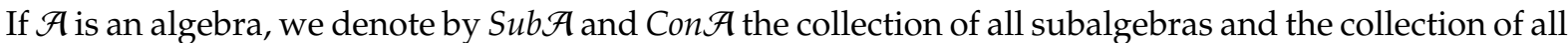
congruences of $\mathcal{A}$, as well as the corresponding lattices under set inclusion. They are called the subalgebra lattice and the congruence lattice of $\mathcal{A}$.

The following well known theorem gives a connection between the congruence lattice of an algebra and the lattice of all equivalences on the support of that algebra:

Theorem 2.1. ([1]) The congruence lattice of an algebra is a complete sublattice of the lattice of all equivalence relations on the support of that algebra.

A weak congruence relation on an algebra $\mathcal{A}=(A, H)$ is a relation $\varrho$ on $A$ that is symmetric, transitive, compatible with all the operations of $\mathcal{A}$ (operations in $H$ ) and weakly reflexive (for every nullary operation $c \in H, c \varrho c)$.

The collection $C w \mathcal{A}$ of weak congruences on an algebra $\mathcal{A}$ is an algebraic lattice under inclusion.

In the weak congruence lattice, diagonal relation on the algebra and on all the subalgebras play an important role:

$$
\begin{aligned}
& \Delta=\{(x, x) \mid x \in A\}, \\
& \Delta_{B}=\{(x, x) \mid x \in B\}, \text { for any } \mathcal{B} \text { subalgebra of } \mathcal{A} .
\end{aligned}
$$

Throughout the paper will also use the notation: if $\mathcal{B}$ is a subalgebra of $\mathcal{A}$ and $\theta$ is a congruence on $\mathcal{A}$,

$$
B[\theta]=\{x \in A \mid x \theta y \text { for some } y \in B\} .
$$

The weak congruence lattice is important in structure investigations of algebras, since it contains other known lattices connected with algebras:

If $C w \mathcal{A}$ is the lattice of weak congruences of an algebra $\mathcal{A}$, then:

(i) for every subalgebra $\mathcal{B}$ of $\mathcal{A}, \operatorname{Con} \mathcal{B}$ is the interval sublattice $\left[\Delta_{B}, B^{2}\right]$, in particular $\operatorname{Con} \mathcal{A}=\uparrow \Delta$;

(ii) the lattice $\operatorname{Sub} \mathcal{A}$ of subuniverses of $\mathcal{A}$ is isomorphic with the principal ideal $\downarrow \Delta$, under $\mathcal{B} \mapsto \Delta_{B}$;

(iii) the map $m_{\Delta}: \varrho \mapsto \varrho \wedge \Delta$ is a homomorphism from $C w \mathcal{A}$ onto $\downarrow \Delta$.

The homomorphism in (iii) defines a congruence relation on the weak congruence lattice, every class of which is the set of congruences of a subalgebra $\mathcal{B}$ of $\mathcal{A}$, i.e. the interval $\left[\Delta_{B}, B^{2}\right]$ of the weak congruence lattice. The set of classes forms a lattice isomorphic to $S u b \mathcal{A}$.

The problem of representation of an algebraic lattice by the weak congruence lattice of an algebra is given in [8]: Let $\mathcal{L}=(L, \vee, \wedge)$ be an algebraic lattice and $a \in L$. Is there an algebra such that its weak congruence lattice is isomorphic with $\mathcal{L}$, the diagonal relation being the image of a under the isomorphism?

If the answer is 'yes', we say that $a$ is a $\Delta$-suitable element of lattice $\mathcal{L}$.

Since $\Delta$ is codistributive in $C w \mathcal{A}$, i.e., for all $\rho, \theta \in C w \mathcal{A}$, we have

$$
\Delta \wedge(\rho \vee \theta)=(\Delta \wedge \rho) \vee(\Delta \wedge \theta)
$$

(see e.g. ([8])), we have that every $\Delta$-suitable element of a lattice is codistributive.

A codistributive element of an algebraic lattice fulfills the following:

Theorem 2.2. ([8]) If an element of an algebraic lattice $\mathcal{L}=(L, \vee, \wedge)$ is codistributive, then for every $b \in \downarrow a$, the family $\{x \in L \mid a \wedge x=b\}$ has the top element. 
If $\mathcal{L}$ is an algebraic lattice and $x \in L$, we denote the top element of the family $\{y \in L \mid a \wedge y=a \wedge x\}$ by $\bar{x}$.

Some further conditions, which a codistributive element of an algebraic lattice must fulfill in order to be $\Delta$-suitable, are given in the following proposition, and they are based on the properties of the weak congruence lattice:

Proposition 2.3. ([8]) $A \Delta$-suitable element $a \in L$ satisfies the following:

(1) if $x \wedge y \neq \mathbf{0}$ then $\overline{x \vee y}=\bar{x} \vee \bar{y}$;

(2) if $\bar{x} \neq \mathbf{0}$ and $\bar{x}<y$, then $\overline{y \wedge a} \neq y \wedge a$;

(3) if $x<a$ and $\bar{x} \neq 0$, then $\bigvee\{y \in \uparrow a \mid y \vee \bar{x}<\mathbf{1}\} \neq \mathbf{1}$;

(4) If $y \in \downarrow a$ and $x<y$, then there exists $z \in[y, \bar{y}]$, such that

- for all $t \in[x, \bar{x}]$, the set $\left\{c \in \operatorname{Ext}^{y}(t) \mid c \leqslant z\right\}$ is either empty or has the top element, and

- for all $t \in[x, \bar{x}]$, the set $\left\{c \in \operatorname{Ext}^{y}(t) \mid c \nless z\right\}$ is an antichain (possibly empty), where

$$
\operatorname{Ext}^{y}(t):=\{w \in[y, \bar{y}] \mid w \cap \bar{x}=t\} .
$$

In the next section, conditions (3) and (4) from Proposition 2.3 will be generalized.

\section{Results}

We start from the lemma that we will use for further results.

Lemma 3.1. If $a$ is a $\Delta$-suitable element of a lattice $\mathcal{L}$, and $b \leqslant a$, then $b$ is a $\Delta$-suitable element of the ideal $\downarrow \bar{b}$.

Proof. If $\bar{b}=0$, then the assertion of the lemma follows immediately, so let $\bar{b} \neq 0$. Let $\mathcal{A}=(A, H)$ be an algebra such that $C w \mathcal{A}$ is isomorphic to $\mathcal{L}$ under a lattice isomorphism $\pi$, mapping $\Delta$ to $a$. Since $b \leqslant a, b$ corresponds to $\Delta_{B}$, for a nonempty subalgebra $\mathcal{B}=(B, H)$ of algebra $\mathcal{A}$. Now $C w \mathcal{B}$ is isomorphic to $\downarrow \bar{b}$ under the restriction of the isomorphism $\pi$ to $B$ that maps the diagonal relation $\Delta_{B}$ to $b$. Therefore, $b$ is $\Delta$-suitable in the lattice $\downarrow \bar{b}$.

The next proposition is a generalization of condition (3) in Proposition 2.3.

Proposition 3.2. If $a$ is a $\Delta$-suitable element of a lattice $\mathcal{L}$ and $x, y \in L$, such that $x<y \leqslant a$ and $\bar{x} \neq 0$, then the interval $[\bar{x}, \bar{y})$ has the top element.

Proof. If $(\bar{x}, \bar{y})=\emptyset$, then $[\bar{x}, \bar{y})=\bar{x}$, and $\bar{x}$ is the top element of the interval. Therefore let $(\bar{x}, \bar{y}) \neq \emptyset$ and let $t \in(\bar{x}, \bar{y})$. Since $x=a \wedge \bar{x} \leqslant a \wedge t \leqslant \bar{y} \wedge a=y$, we have $x \leqslant t \wedge a \leqslant y$. Since $x<y$, we have $t \wedge a=x$ or $t \wedge a=y$. If $t \wedge a=x$, then $t \leqslant \bar{x}$, which is impossible, so $t \wedge a=y$ and $t \in[y, \bar{y}]$. Since $t \vee \bar{x}=t<\bar{y}$, we have: $t \in\{z \in \uparrow y \mid z \vee \bar{x}<\bar{y}\}$ and $\{z \in \uparrow y \mid z \vee \bar{x}<\bar{y}\} \neq \emptyset$.

According to the previous lemma, $y$ is $\Delta$-suitable in the lattice $\downarrow \bar{y}$. Since $x<y, \bar{x} \neq 0$, by condition (3) in Proposition 2.3 we have:

$$
\bigvee\{z \in \uparrow y \mid z \vee \bar{x}<\bar{y}\} \neq \bar{y}
$$

Notice that $\bigvee\{z \in \uparrow y \mid z \vee \bar{x}<\bar{y}\} \geqslant \bigvee[\bar{x}, \bar{y})$, since $\{z \in \uparrow y \mid z \vee \bar{x}<\bar{y}\} \supset[\bar{x}, \bar{y}) \cap \uparrow y$. Therefore

$$
\bigvee[\bar{x}, \bar{y}) \leqslant \bigvee\{z \in \uparrow y \mid z \vee \bar{x}<\bar{y}\}<\bar{y}
$$

and $\bigvee[\bar{x}, \bar{y}) \in[\bar{x}, \bar{y})$. Thus $[\bar{x}, \bar{y})$ has the top element.

Remark 3.3. Condition from Proposition 3.2 is a generalization of condition (3) in Proposition 2.3. 
Indeed, if $x<a$ and Proposition 3.2 holds, then $[\bar{x}, 1)$ has the top element $t$. If $t=\bar{x}$, then $y \vee \bar{x}=1$ for all $y \in \uparrow a$ and the set $\{y \in \uparrow a \mid y \vee \bar{x}<1\}$ is empty and $\bigvee\{y \in \uparrow a \mid y \vee \bar{x}<1\}=0<1$. If $t>\bar{x}, t$ is greater than or equal to $\bigvee\{y \in \uparrow a \mid y \vee \bar{x}<1\}$, since for every $s \in\{y \in \uparrow a \mid y \vee \bar{x}<\mathbf{1}\}$ we have: $s \vee \bar{x} \in[\bar{x}, 1)$, and so for every element of $\{y \in \uparrow a \mid y \vee \bar{x}<\mathbf{1}\}$, there is an element of $[\bar{x}, 1)$ greater than or equal to it. So $\bigvee\{y \in \bar{a} \mid y \vee \bar{x}<1\} \leqslant \bigvee[\bar{x}, 1)=t<1$ and the condition (3) holds.

The condition in the previous proposition is more general, because it cannot be derived from conditions (1)-(4) of Theorem 2.3, which is illustrated in the following example.

Example 3.4. A lattice and an element $a$ are given in Figure 1 .

Element $a$ fulfills the conditions of Theorem 2.3, but not the condition in Proposition 3.2. Therefore, we conclude that element $a$ in Figure 1 is not $\Delta$-suitable by the new condition.

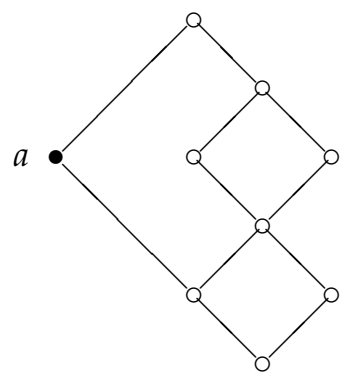

Figure 1

The previous proposition is further generalized in the following theorem.

Theorem 3.5. If $a$ is a $\Delta$-suitable element of a lattice $\mathcal{L}, x, y \in L, \bar{x} \neq 0$ and $x<y \leqslant a$, then

$$
[y \vee \bar{x}, \bar{y}) \backslash \bigcup_{z \in(x, y)}[y \vee \bar{z}, \bar{y})
$$

is either the empty set, or has the top element.

Proof. Let $\mathcal{A}$ be an algebra whose weak congruence lattice is isomorphic to $\mathcal{L}$ under an isomorphism mapping $a$ to $\Delta_{A}$. The isomorphism maps $x, y, z$ to $\Delta_{B}, \Delta_{C}$ and $\Delta_{D}$, for some nonempty subuniverses $B, C$ and $D$ of the algebra $\mathcal{A}$.

Suppose $[y \vee \bar{x}, \bar{y}) \backslash \bigcup_{z \in(x, y)}[y \vee \bar{z}, \bar{y}) \neq \emptyset$; then the corresponding subset of $C w \mathcal{A}$ is not empty, i.e.,

$$
\left[\Delta_{C} \vee B^{2}, C^{2}\right) \backslash \bigcup_{D \in(B, C)}\left[\Delta_{C} \vee D^{2}, C^{2}\right) \neq \emptyset .
$$

Let $\varrho \in\left[\Delta_{C} \vee B^{2}, C^{2}\right) \backslash \bigcup_{D \in(B, C)}\left[\Delta_{C} \vee D^{2}, C^{2}\right)$. Clearly $\varrho \in \operatorname{Con} C$. If $B[\varrho]=\{x \in C \mid x \varrho y$ for some $y \in B\}$, it is a subuniverse of $C$ containing $B$, i.e. $B \leqslant B[\varrho] \leqslant C$.

If $s, t \in B[\varrho]$, we have $s \varrho p$ and $t \varrho r$ for some $p, r \in B$. Since $\varrho \supset B^{2}$, we have also $p \varrho r$, and so $(s, t) \in \varrho$. Since this is true for all $s, t \in B[\varrho]$, we have $\varrho \supseteq B[\varrho]^{2}$. But $\varrho \nsupseteq D^{2}$ for any $D \in(B, C]$, so $B[\varrho]=B$ and $\varrho \subseteq B^{2} \cup(C \backslash B)^{2}$. Since this is true for any $\varrho \in\left[\Delta_{C} \vee B^{2}, C^{2}\right) \backslash \bigcup_{D \in(B, C)}\left[\Delta_{C} \vee D^{2}, C^{2}\right)$ and $B^{2} \cup(C \backslash B)^{2}$ is an equivalence on $B$, the supremum $\theta$ of this set in the set of all equivalences on $C$ is also a subset of $B^{2} \cup(C \backslash B)^{2}$. By Theorem 2.1, we have also that $\theta$ is also the supremum of $\left[\Delta_{C} \vee B^{2}, C^{2}\right) \backslash \bigcup_{D \in(B, C)}\left[\Delta_{C} \vee D^{2}, C^{2}\right)$ in the set of all congruences of $C$. As $\theta \subseteq B^{2} \cup(C \backslash B)^{2}$, we have $B[\theta]=B$. Now it is easy to see that $\theta \in\left[\Delta_{C} \vee B^{2}, C^{2}\right) \backslash \bigcup_{D \in(B, C)}\left[\Delta_{C} \vee D^{2}, C^{2}\right)$, 
because any element of $\left[\Delta_{C} \vee B^{2}, C^{2}\right)$ contains $\Delta_{C} \vee B^{2}$ and so does $\theta$; also $\theta \subseteq B^{2} \cup(C \backslash B)^{2}$ and so $\theta<C^{2}$ and $\theta \in\left[\Delta_{C} \vee B^{2}, C^{2}\right)$. Since $\theta \subseteq B^{2} \cup(C \backslash B)^{2}$, we have that $\theta \nsupseteq D^{2}$ for any $D \in(B, C)$ and so $\theta \notin\left[\Delta_{C} \vee D^{2}, C^{2}\right)$ for any $D \in(B, C)$. Thus $\theta \in\left[\Delta_{C} \vee B^{2}, C^{2}\right) \backslash \bigcup_{D \in(B, C)}\left[\Delta_{C} \vee D^{2}, C^{2}\right)$, and $\theta$ is the top element of the set. Therefore, the corresponding subset of $L$ has the top element.

Remark 3.6. It is straightforward that the condition from Proposition 3.2 is a special case of the condition from Theorem 3.5.

The next example shows that the condition from Theorem 3.5 is more general than other conditions.

Example 3.7. An element $a$ in the lattice in Figure 2 fulfills all the conditions from Proposition 2.3, as well as the condition in Proposition 3.2, but it is not $\Delta$-suitable, because the condition of Theorem 3.5 does not hold.

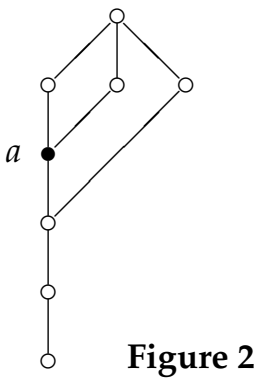

In the following part, we give a generalization of condition (4) of Proposition 2.3, which is at the same time a generalization of Proposition 3.2.

We use the notation and ideas from the proof of Theorem 2.22 from ([8]) by M. Ploščica.

Lemma 3.8. Let $\mathcal{A}$ be an algebra and $\mathcal{B}$ and $\mathcal{C}, \mathcal{B} \leq \mathcal{C}$ be two subalgebras of $\mathcal{A}$. Then the collection

$$
\Gamma^{B, C}=\{\rho \in \operatorname{Con} C \mid \text { there is no } x \in B, y \in C \backslash B \text { such that } x \rho y\}
$$

has the supremum.

Proof. $\Gamma^{B, C}$ is non-empty, since $\Delta_{C} \in \Gamma^{B, C}$. Let $\gamma$ be the supremum of $\Gamma^{B, C}$ in ConC. It belongs to $\Gamma^{B, C}$ since, by Theorem 2.1, it coincides with the the supremum of $\Gamma^{B, C}$ in the lattice of all equivalences on $C$, and the latter supremum is less or equal to $B^{2} \cup(C \backslash B)^{2}$.

Proposition 3.9. If a be $a \Delta$-suitable element of a lattice $\mathcal{L}$, then if $x, y \in \downarrow a, \bar{x} \neq 0$ and $x<y$, there exists $z \in[y, \bar{y})$, such that

- for all $t \in[x, \bar{x}]$, the set $\left\{c \in \operatorname{Ext}^{y}(t) \mid c \leqslant z\right\}$ is either empty or has the top element, and

- for all $t \in[x, \bar{x}]$, the set $\left\{c \in \operatorname{Ext}^{y}(t) \mid c \nless z\right\}$ is an antichain (possibly empty), where

$$
\operatorname{Ext}^{y}(t):=\{w \in[y, \bar{y}] \mid w \cap \bar{x}=t\}
$$

Proof. Since $a$ is a $\Delta$-suitable element, then there is an algebra $\mathcal{A}$ whose weak congruence lattice is isomorphic to $\mathcal{L}$ under an isomorphism $f$ mapping $a$ to $\Delta_{A}$. From $x, y \in \downarrow a$, it follows that there are subalgebras $\mathcal{B}$ and $C$, such that $\Delta_{B}$ and $\Delta_{C}$ correspond to $x$ and $y$ under $f$, respectively and $\mathcal{B}<C$ in lattice $S u b \mathcal{A}$. Let $\Gamma^{B, C}$ be the collection of congruences from Con $C$ defined in Lemma 3.8. By Lemma 3.8, $\Gamma^{B, C}$ has the supremum $\gamma$. Since $\gamma \in \Gamma^{B, C}$ then we have $\gamma \leqslant B^{2} \cup(C \backslash B)^{2}<C^{2}$ and it belongs to ConC $\backslash\left\{C^{2}\right\}=\left[\Delta_{C}, C^{2}\right)$. 
$\gamma$ would be a relation that corresponds to element $z$ in the proposition. Let $\alpha \in \operatorname{Con} \mathcal{B}$, and we consider a collection of relations $\Upsilon=\left\{\rho \in \operatorname{Ext}^{C}(\alpha) \mid \rho \leqslant \gamma\right\}$. Suppose that this collection is not empty. For every $\beta \in \operatorname{Ext}^{C}(\alpha)$, it follows $\beta \cap B^{2}=\alpha$, and since $\beta \leqslant \gamma$, we have that every $\varrho \in \Upsilon$ belongs to $\Gamma^{B, C}$. Then, $\bigvee \Upsilon \in \Gamma^{B, C}$ and $\bigvee \Upsilon \cap B^{2}=\alpha$. $\bigvee \Upsilon$ is the required top element.

To prove the second part of the proposition, suppose that $\alpha \in \operatorname{Con} \mathcal{B}$ and set $\Phi=\left\{\varrho \in \operatorname{Ext}^{C}(\alpha) \mid \varrho \nless \gamma\right\}$ is not empty. If $\alpha_{1}, \alpha_{2} \in \Phi$, then $\alpha_{1} \cap B^{2}=\alpha_{2} \cap B^{2}=\alpha$. Since $\alpha_{1} \nless \gamma$ and $\alpha_{1} \nless \gamma$, it follows that there are $x_{1}, x_{2} \in B, y_{1}, y_{2} \in C \backslash B$, such that $x_{1} \alpha_{1} y_{1}$ and $x_{2} \alpha_{2} y_{2}$. Hence $B\left[\alpha_{1}\right]=C$, and $B\left[\alpha_{2}\right]=C$, since $B\left[\alpha_{1}\right]$ and $B\left[\alpha_{1}\right]$ are subalgebras of $C$, and $\mathcal{B}<C$. If $\alpha_{1} \subsetneq \alpha_{2}$, let $(x, y) \in \alpha_{2} \backslash \alpha_{1}$. There exist $x_{0}, y_{0} \in B$, such that $x_{0} \alpha_{1} x$ and $y_{0} \alpha_{1} y$ and, consequently $x_{0} \alpha_{2} x$ and $y_{0} \alpha_{2} y$. Now, clearly $\left(x_{0}, y_{0}\right) \in \alpha_{2} \backslash \alpha_{1}$, and $\alpha_{2} \cap B^{2} \neq \alpha_{1} \cap B^{2}$, and we have a contradiction. Therefore, $\alpha_{1}$ and $\alpha_{2}$ are not comparable under $\subset$ and $\Phi$ is an antichain.

Remark 3.10. This is a generalization of condition (4) of Proposition 2.3, except for the trivial case when $\bar{x}=0$. Indeed, in condition (4) we have $z \in[y, \bar{y}]$ and here $z \in[y, \bar{y})$.

The assertion of Proposition 3.2 also follows from the previous proposition: Let $x<y \leqslant a \in L$ and $z \in[y, \bar{y})$ the element, existing by the previous proposition. The set $(\bar{x}, \bar{y}]=[y \vee \bar{x}, \bar{y}]=\operatorname{Ext} t^{y}(\bar{x})$ is either equal to $\{\bar{y}\}$, or has at least two comparable elements (because $\bar{y} \in \operatorname{Ext} t^{y}(\bar{x})$ ). In the first case $[\bar{x}, \bar{y})=\{\bar{x}\}$ and the assertion of Proposition 3.2 follows. In case $E x t^{y}(\bar{x})$ contains two comparable elements, from the previous proposition follows that one of them must be less or equal to $z$, so let $u \leqslant z$ be one of them. Now $\bar{x} \geqslant z \wedge \bar{x} \geqslant u \wedge \bar{x}=\bar{x}$, so $z \wedge \bar{x}=\bar{x}$, i.e. $z \in \operatorname{Ext} t^{y}(\bar{x})$.

Now, $z$ is the top element of $\operatorname{Ext} t^{y}(\bar{x}) \backslash\{\bar{y}\}:$ if $s \in E x t^{y}(\bar{x}) \backslash\{\bar{y}\}$, by the previous proposition we have $s \leqslant z$, since the set $\left\{c \in \operatorname{Ext}^{y}(\bar{x}) \mid c \nless z\right\}$ already contains $\bar{y}$ and $z<\bar{y}$. Since $[\bar{x}, \bar{y})=\{x\} \cup\left(\operatorname{Ext} t^{y}(\bar{x}) \backslash\{\bar{y}\}\right), z$ is also the top element of $[\bar{x}, \bar{y})$.

A generalization of Proposition 3.9 is given in the following theorem:

Theorem 3.11. If $a$ is a $\Delta$-suitable element of a lattice $\mathcal{L}=(L, \vee, \wedge)$ and $x, y \in L$, such that $\bar{x} \neq 0, x<y \leqslant a$, then there is a mapping $\varphi:[x, y) \rightarrow[y, \bar{y})$, such that:

- for all $t \in[x, \bar{x}]$ and $u \in[x, y)$, the set $\left\{c \in \operatorname{Ext}^{y}(t) \mid c \leqslant \varphi(u)\right\}$ is either empty or has the top element, and

- for all $t \in[x, \bar{x}]$, the set $\left\{c \in \operatorname{Ext}^{y}(t) \mid(\forall u \in[x, y))(c \nless \varphi(u))\right\}$ is an antichain (possibly empty), where

$$
\operatorname{Ext}^{y}(t):=\{w \in[y, \bar{y}] \mid w \cap \bar{t}=t\} .
$$

Proof. Let $\mathcal{A}$ be an algebra representing lattice $\mathcal{L}$ together with element $a$, i.e. let $\mathcal{A}$ be an algebra isomorphic

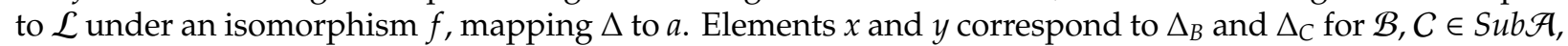
and the interval $[B, C)$ of $S u b \mathcal{A}$ is

$$
[B, C)=\left\{A_{i} \mid i \in I\right\} .
$$

For $i \in I$ let $\Gamma^{A_{i}, C}$ be the subset of $C o n C$ defined as follows:

$\Gamma^{A_{i}, C}=\left\{\varrho \in \operatorname{Con} C \mid \varrho \subseteq A_{i}^{2} \cup\left(C \backslash A_{i}\right)^{2}\right\}$.

Let $\gamma_{i}$ be the supremum of $\Gamma^{A_{i}, C}$ in lattice ConC for $i \in I$ (which exists by Lemma 3.8) and $\gamma_{i} \neq C^{2}$ for $i \in I$. Now, we define mapping $\bar{\varphi}:[B, C) \rightarrow C o n C$, by:

$$
\bar{\varphi}\left(A_{i}\right)=\gamma_{i}
$$

and the required mapping $\varphi:[x, y) \rightarrow[y, \bar{y})$ is defined by

$$
\varphi=f^{-1} \circ \bar{\varphi} \circ f .
$$

Let $\varrho$ be a congruence of $C$. We have the following:

(i) $\varrho \in \Gamma^{A_{i}, C} \Rightarrow \varrho \leqslant \gamma_{i}$ for $i \in I$;

(ii) $\left[(\forall i \in I) \rho \notin \Gamma^{A_{i}, C}\right] \Rightarrow B[\rho]=C$. 
The assertion (ii) follows from the fact that $B[\rho]$ is a subuniverse of $C$ containing $B$, i.e. it is equal to $A_{i}$ for some $i \in I$ or $B[\varrho]=C$. If $B[\varrho]=A_{i}$, then $\varrho \subseteq A_{i}^{2} \cup\left(C \backslash A_{i}\right)^{2}$ and thus $\varrho \in \Gamma^{A_{i}, C}$. Therefore $B[\varrho]=C$

Now, let $\phi \in \operatorname{Con} \mathcal{B}$, and $\theta \in \operatorname{Ext}^{\mathrm{C}}(\phi)$. If $B[\theta]=A_{i}$, then $\theta \in \Gamma^{A_{i}, C}$ and $\theta \leqslant \gamma_{i}$. By the same argument as used above we have that $\varrho=\bigvee\left\{\theta \in E x t^{C}(\phi) \mid \theta \leqslant \gamma_{i}\right\} \in \Gamma^{A_{i}, C}$. We also have that $\varrho \in E x t^{C}(\phi)$, so

$$
\left\{\theta \in \operatorname{Ext}^{\mathrm{C}}(\phi) \mid \theta \leqslant \gamma_{i}\right\}
$$

has the top element $\tau_{i}$.

Let $\varrho, \psi \in\left\{\tau \in \operatorname{Ext}^{C}(\phi) \mid(\forall i \in I)\left(\tau \nless \gamma_{i}\right)\right\}$. By (ii) we have that $B[\varrho]=B[\psi]=C$ If $\varrho<\psi$ and $(d, e) \in \psi \backslash \varrho$, then $b \varrho d$ and $c \varrho e$ for some $b, c \in B$. Now from $b \psi d$ and $c \psi e$ (by $d \psi e$ ) it follows that $b \psi c$. If $(b, c) \in \varrho$, then $(d, e) \in \varrho$, so $(b, c) \notin \varrho$ and $\varrho \cap B^{2} \neq \psi \cap B^{2}$, which contradicts the assumptions. Thus, any two elements of

$$
\left\{\tau \in \operatorname{Ext}^{C}(\phi) \mid(\forall i \in I)\left(\tau \nless \gamma_{i}\right)\right\}
$$

are incomparable. The assertion of the theorem follows.

We give an example in the sequel proving that the condition from Theorem 3.11 is a generalization of the previously introduced conditions.

Example 3.12. Element $a$ of the lattice in Figure 3 fulfills all the conditions of Propositions 2.3,3.2,3.9, as well as of Theorem 3.5, but it is not $\Delta$-suitable, because it does not fulfill the condition of Theorem 3.11:

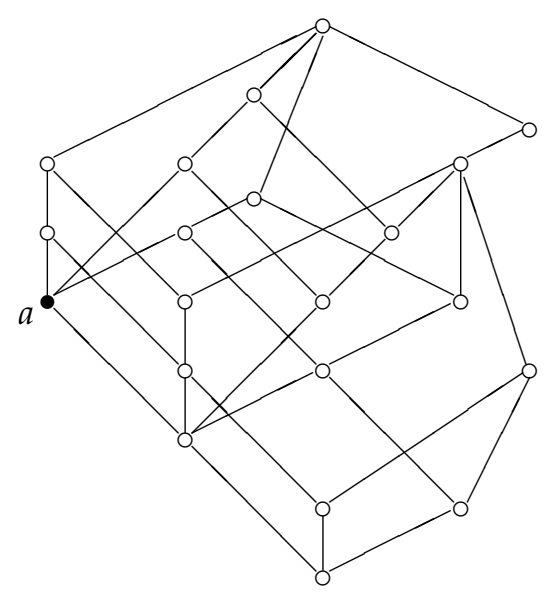

Figure 3

After some generalizations of the known criteria for a $\Delta$-suitable element, we can summarize these results in the following theorem:

Theorem 3.13. A $\Delta$-suitable element $a \in L$ satisfies the following:

(1) if $x \wedge y \neq \mathbf{0}$, then $\overline{x \vee y}=\bar{x} \vee \bar{y}$;

(2) if $\bar{x} \neq \mathbf{0}$ and $\bar{x}<y$, then $\overline{y \wedge a} \neq y \wedge a$;

(3) If $\bar{x} \neq 0$ and $x<y \leqslant a$, then $[y \vee \bar{x}, \bar{y}) \backslash \bigcup_{z \in(x, y)}[y \vee \bar{z}, \bar{y})$ is either the empty set, or has the top element;

(4) If $\bar{x} \neq 0, x<y \leqslant a$, then there is a mapping $\varphi:[x, y) \rightarrow[y, \bar{y})$, such that: 
- for all $t \in[x, \bar{x}]$ and $u \in[x, y)$, the set $\left\{c \in \operatorname{Ext}^{y}(t) \mid c \leqslant \varphi(u)\right\}$ is either empty or has the top element, and - for all $t \in[x, \bar{x}]$, the set $\left\{c \in \operatorname{Ext}^{y}(t) \mid(\forall u \in[x, y))(c \nless \varphi(u))\right\}$ is an antichain (possibly empty), where

$$
\operatorname{Ext}^{y}(t):=\{w \in[y, \bar{y}] \mid w \cap \bar{t}=t\}
$$

Remark 3.14. This theorem includes all the known criteria, given in Proposition 2.3: condition (3) is a generalization of Proposition 3.2 and therefore a generalization of condition (3) of Proposition 2.3; condition (4) is an obvious generalization of Proposition 3.9, and accordingly a generalization of condition (4) of Proposition 2.3.

Remark 3.15. The question arises whether these four conditions are independent. The lattice in Figure 3 proves that condition (4) cannot be derived from other three. The lattice in Figure 2 proves that condition (3) is independent. In the next example we provide lattices that prove independence of conditions (1) and (2).

Example 3.16. The lattice in Figure 4 fulfills conditions (1), (3) and (4) of the previous theorem, but does not fulfill condition (2).

The lattice in Figure 5 fulfills conditions (2), (3) and (4) of the previous theorem, but does not fulfill condition (1).

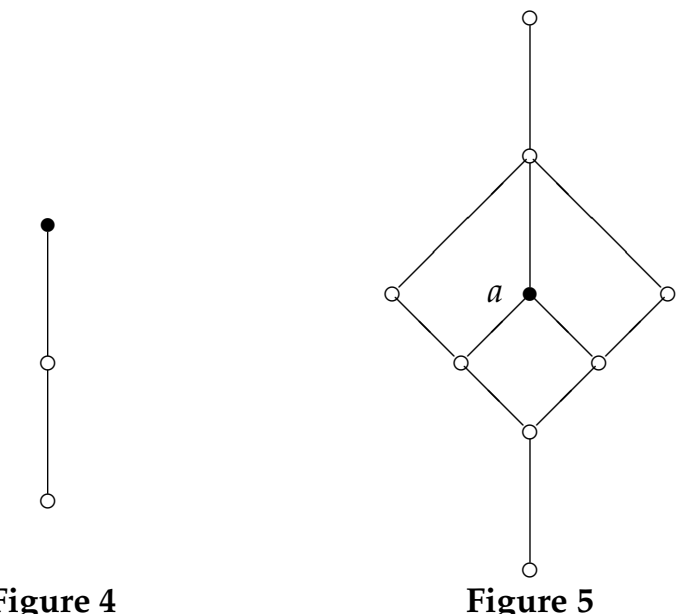

\section{References}

[1] S. Burris, H.P. Sankappanavar, A Course in Universal Algebra, Springer-Verlag, New York, 1981.

[2] G. Czedli, B. Šešelja, A. Tepavčević: Semidistributive elements in lattices; application to groups and rings, Algebra Univers. 58 (2008) 349-355.

[3] G. Czédli, M. Erné, B. Šešelja, A. Tepavčević, Characteristic triangles of closure operators with applications in general algebra, Algebra Univers. 62(4) (2009) 399-418.

[4] B. A. Davey, H. A. Priestley, Introduction to Lattices and Order, Cambridge University Press 1990.

[5] G. Grätzer, General Lattice Theory, Second edition, Birkhäuser Verlag 2003.

[6] W.A. Lampe, Results and problems on congruence lattice representations, Algebra univers. 55 (2006) 127-135.

[7] M. Ploščica, Graphical compositions and weak congruences, Publ. Inst. Math. Beograd 56(70) 1994 34-40.

[8] B. Šešelja, A. Tepavčević, Weak Congruences in Universal Algebra, Institute of Mathematics, Novi Sad, 2001.

[9] B. Šešelja, A. Tepavčević, A Note on CIP Varieties, Algebra Univers. 45 (2001) 349-351.

[10] B. Šešelja, A. Tepavčević, On Weak Congruence Lattices Representation Problem, Proc. of the 10th Congress of Yugoslav Mathematicians, Belgrade, 2001, 177-184.

[11] A. Tepavčević, Diagonal relation as a continuous element in a weak congruence lattice, Proc. of the International Conference General Algebra and Ordered Sets, Olomouc, 1994, 156-163.

[12] G. Vojvodić, B. Šešelja, On the lattice of weak congruence relations, Algebra Univers. 25 (1988) 121-130.

[13] G. Vojvodić, B. Šešelja, The diagonal relation in the lattice of weak congruences and the representation of lattices, Rev. of Res. Fac. Sci., Univ. Novi Sad 19(1) (1989) 167-178. 